\title{
Le harcèlement de rue et la thèse du continuum des violences
}

\author{
Carole GAYET-VIAUD
}

CNRS-CESDIP, EHESS-CEMS 
Le problème public du harcèlement de rue s'est aujourd'hui imposé dans le débat public hexagonal, en s'adossant à deux notions clés: celle de «harcèlement» et celle de «continuum des violences». La notion de harcèlement désigne, dans le champ des études sur les violences, mais aussi dans le droit français ${ }^{[1]}$, une forme de violence qui peut être aussi bien psychologique, verbale que physique, et qui s'exerce de façon répétée sur une victime. La notion de continuum des violences renvoie, quant à elle, à un plan analytique d'interprétation des formes et degrés divers de violence. C'est dans le champ des études féministes que la formule a été forgée (Kelly, 2019) ${ }^{[2]}$ pour affirmer l'existence d'un lien entre les différentes formes de violence et inviter à considérer avec prudence les nomenclatures qui les distinguent trop nettement entre elles, jusqu'à les cloisonner, en fonction d'indicateurs de gravité a priori Ces deux notions, désormais mobilisées de concert par les milieux académiques, militants et politiques ${ }^{[3]}$, se prêtent, derrière leur omniprésence et l'apparent consensus que celle-ci suggère, à une diversité d'usages et d'interprétations. Le succès médiatique et politique, et plus récemment, le devenir législatif des raisonnements qu'elles ont contribué à porter, justifient qu'on les envisage depuis les usages auxquels elles ont donné lieu et les conséquences qu'elles ont permis de produire ${ }^{[4]}$.

On propose de revenir dans cet article sur la genèse de la théorisation des rapports femmes-hommes en public, et plus spécifiquement des problèmes posés par les "propos publics offensants ${ }^{[5]}$ " subis par les femmes dans les espaces publics urbains, en termes de harcèlement de rue. On montrera comment cette thématisation a contribué au processus de politisation de la question; on identifiera ensuite des effets inaperçus de cette configuration du phénomène (Gusfield, 2009) et certaines implications des notions de «continuum de violence» et de «harcèlement». On s'appuiera sur une comparaison avec les théories de

1 En France la création du délit de harcèlement sexuel date de 1992, la loi pénale ayant été mise en œuvre en 1994, modifiée à plusieurs reprises, supprimée, avant d'être rétablie par la loi $n^{\circ} 2012-954$ du 6 août 2012.

2 On revient de façon détaillée sur cette genèse dans la suite du présent article.

3 Les notions sont au centre d'un nombre croissant de publications académiques (Bathelier et al., 2017; Delage et al., 2019) et de rapports publics: rapports d'information de l'Assemblée nationale et du sénat, rapports d'étude du Centre Hubertine Auclert (2018), de I'IAU (2016), du HCEf/h (2015).

4 L'analyse porte ici sur la mobilisation des concepts de harcèlement et de continuum de violence, dans la formation d'une théorie du harcèlement de rue et dans l'élaboration de stratégies politiques visant à le pénaliser. La réflexion entend donc mettre au jour la logique et la rhétorique qui président à l'élaboration (et à la justification) des politiques pénales dédiées au harcèlement de rue en France, sans prétendre ici en étudier les effets pratiques, dans les politiques publiques effectivement menées (peu documentées au moment où ce texte est écrit). On entend ainsi contribuer à l'étude sociologique de la "culture des problèmes publics" (Gusfield, 2009).

5 Voir, dans ce numéro, la traduction de I'article de Laura Beth Nielsen. 


\section{Enquête et matériau}

Les analyses qui suivent s'appuient sur des matériaux assemblés au fil de plusieurs enquêtes:

1) Une enquête doctorale conduite en région parisienne entre 2000 et 2007 portant sur la civilité dans les interactions urbaines, reposant sur l'observation directe prolongée des espaces publics urbains de quatre secteurs de la capitale (rues, transports publics, jardins, cafés et espaces commerciaux ouverts au public) complétée par des conversations informelles et la conduite d'entretiens approfondis (une trentaine), le recueil de récits portant sur l'expérience des interactions urbaines de première main et de seconde main (forums, témoignages sur des plateformes numériques).

2) Une enquête, menée de 2012 à 2018, portant sur les incivilités, leur devenir problème public, les politiques, les métiers et pratiques de régulation qui les visent, consistant dans l'étude ethnographique de deux quartiers du nord parisien, ainsi que deux quartiers d'une grande ville du sud de la France, ces sites comportant à chaque fois un quartier mixte de centre urbain et un quartier "politiques de la ville" (QPV) classé "zone de sécurité prioritaire" (ZSP). Dans ce cadre, ont été réalisées, d'une part des analyses documentaires (corpus de doléances orales et écrites), des observations participantes, des observations directes, et d'autre part, des entretiens auprès de professionnels parmi une diversité d'acteurs de la vie urbaine et de la sécurité publique $(n=105)$ : sociétés de transport, bailleurs, responsables sécurité des villes, délégués du préfet, chargés de mission cohésion sociale, policiers (chefs de service, responsables de BST [brigades spécialisées de terrain] et référents scolaires), associations de prévention spécialisée, associations de médiation, élus, habitants et responsables d'associations (de quartier, de retraités, de parents d'élèves, de jardin partagé, de femmes du quartier).

3) À ces matériaux s'ajoutent ceux collectés dans le cadre de deux sous-enquêtes complémentaires, touchant aux incivilités, à leurs mesures et à leurs régulations: une enquête collective (Maillard et al., 2017) portant sur le dispositif des délégués à la cohésion police-population (DCPP), avec une série d'entretiens conduits auprès de ces policiers retraités réservistes auxquels a été confiée une mission de médiation et de régulation de petits conflits auprès des populations $(n=27)$ et l'analyse des rapports mensuels de deux d'entre eux; une enquête sur les incivilités dans les bibliothèques municipales de la ville de Paris: observations, entretiens menés auprès de bibliothécaires et de cadres de la sécurité publique locale $(n=30)$, et analyse des archives de comptes rendus d'incidents pour la période 2009-2013 ( $n=629)$.

4) Enfin, une enquête collective conduite dans le cadre de plusieurs années de séminaires menés à l'École normale supérieure (ENS) et l'École des hautes études en sciences sociales (EHESS) avec A. Bidet, M. Boutet, F. Chave et E. Le Méner entre 2014 et 2017, portant sur l'ethnographie des engagements, les rapports entre espaces publics urbains et citoyenneté, ayant bénéficié du soutien d'un financement du Labex Tepsis (pour le programme "Ville et citoyenneté»): le matériau collecté a consisté cette fois dans la conduite d'entretiens relatant l'expérience du côtoiement urbain à partir de descriptions de situations d'interactions, de leur perception, des épreuves de tous ordres auxquelles elles donnaient lieu en s'intéressant plus spécifiquement, aux enjeux de la présence et de l'éducation des enfants dans ces contextes $(n=72)$. 
l'incivilité ${ }^{[6]}$ qui ont, elles aussi, dramatisé des nuisances et désordres urbains, auparavant considérés comme négligeables, en les inscrivant dans des dynamiques de plus ample portée. Les incivilités se sont en effet imposées comme objets de politiques publiques au titre, d'une part, des effets produits par leur répétition et leur accumulation; et d'autre part, du fait de leur inscription dans des formes de gradation et d'aggravation, qu'il s'est agi d'enrayer, avec toute la vigueur requise, très tôt en amont. On verra quels enseignements peut offrir cette comparaison, pour éclairer l'orientation présente des politiques publiques visant le harcèlement de rue.

\section{L'émergence du problème public du harcèlement de rue}

\section{La lente politisation}

des rapports de genre

en ville

La notion de «harcèlement de rue» s'est imposée dans le débat public français pour désigner un phénomène qui, s'il n'a suscité l'attention médiatique et politique que récemment, touche à des préoccupations plus anciennes (Bastiat, 2004; Blidon, 2016). En France, l'articulation des questions de genre aux études urbaines a mis du temps à s'imposer. Les travaux pionniers de Jacqueline Coutras (1996) ou de Sylvette Denèfle (2004) jouissent désormais d'une reconnaissance qui ne doit pas faire oublier la tiédeur qui a caractérisé leur réception initiale (Denèfle, Ratouis 2010; Bathelier et al., 2017). C'est aux États-Unis que les premiers travaux visant le phénomène ont été réalisés. Ils ont puisé à deux sources distinctes: les études urbaines héritières de l'École Chicago d'une part, et les études féministes intéressées à la violence de genre d'autre part.

S'agissant de l'entrée urbaine, on trouve chez Erving Goffman (2004, 2013) des descriptions fines des spécificités liées au genre dans l'organisation des relations en public. La performance de genre y est analysée comme vecteur de (sur)exposition des femmes qui occasionne des

6 Les théories ainsi désignées sont celles qui, depuis l'article séminal de Kelling et Wilson (1982), ont proposé, pour expliquer l'insécurité urbaine et la montée de la délinquance, des réflexions donnant un rôle de premier plan aux «incivilités», et ont ainsi inspiré des politiques de sécurité organisées autour de la répression des troubles urbains visibles (notoirement de tout ce qui relève de la petite délinquance de rue), à partir des années 1980 aux États-Unis, et des années 1990 en Europe. 
épreuves redoutées, de réification, de stigmatisation. Pour autant, la spécificité de l'expérience féminine de la vie publique n'est pas pensée de façon systématique, ni considérée comme un objet propre (Gardner, 1980, 1989, 1995). Une telle systématisation n'apparaît qu'avec les travaux pionniers de Carol B. Gardner (qui fut l'étudiante de Goffman ${ }^{[7]}$ ), dont l'approche vise à compléter, corriger et approfondir l'approche goffmanienne des relations en public.

Les recherches qui investissent progressivement ce nouveau domaine d'enquête, à partir des années 1980, s'inscrivent, pour la majorité d'entre elles, dans les perspectives ouvertes par les recherches féministes sur les violences faites aux femmes. Le harcèlement de rue y est saisi comme une déclinaison particulière de la violence de genre, ce qui permet à la fois de le désigner comme forme de violence, et en même temps d'en minorer l'importance (relative), du fait de la priorité accordée au viol et aux violences domestiques. Au sein même des recherches féministes, la question reste ainsi longtemps secondaire, en regard d'une division du travail et d'une hiérarchisation des priorités, à la fois topographique et politique, faisant des violences domestiques et du harcèlement sur le lieu de travail les combats de premier plan, à côté de la lutte pour la criminalisation du viol (Fileborn, Vera-Gray, 2017).

Genèse d'une théorisation:

les travaux pionniers

de Carol B. Gardner sur

le «harcèlement public

des femmes"

Parmi les premières autrices à décrire le phénomène qui deviendra ensuite le harcèlement de rue, figurent la sociologue Carol Brooks Gardner $(1980,1989,1995)$ et l'anthropologue Micaela di Leonardo (1981). Di Leonardo est la première à proposer une définition du phénomène, qu'elle formule dans les termes suivants: il s'agit de tout «événement dans lequel un ou plusieurs inconnu(s) abordent une ou plusieurs femmes dans un lieu public; au travers de regards, de mots, ou de gestes, l'homme affirme son droit de faire intrusion dans l'espace attentionnel de la femme, de la définir comme un objet sexuel, de la forcer à interagir avec lui ${ }^{[8]}{ }$. Cette

7 De même que d'autres figures intellectuelles importantes pour l'étude des relations en public (telles que Lyn Lofland, 1984) ou pour l'étude des questions féministes abordées dans une perspective interactionniste et ethnométhodologique, comme Dorothy Smith (1987). Sur ces filiations, voir Cefaï, 2012

8 Notre traduction. 
définition inscrit le harcèlement de rue dans un rapport hommesfemmes où la sexualisation est imposée, mais dont la source se tient dans les disponibilités différenciées à l'interaction: l'homme peut obliger l'échange, la femme étant par sa seule présence vouée à subir ces infractions attentionnelles.

Gardner conduit ensuite une série d'entretiens approfondis à Indianapolis (1995) qui lui permettent de théoriser ce qu'elle désigne pour sa part comme un "harcèlement public" des femmes. Ses recherches renouvellent la question du côtoiement public, l'analyse des rôles de genre et de la présentation de soi en public en les envisageant depuis une entrée biographique, c'est-à-dire en considérant pour la première fois les temporalités cumulatives qui définissent l'expérience que les femmes font des interactions genrées entre anonymes. La cumulativité se manifeste en particulier dans la récurrence des interactions désagréables que ces rencontres occasionnent, qui s'avèrent constitutives de l'expérience féminine spécifique des rapports en public.

Gardner définit ainsi les femmes comme une catégorie «situationnellement désavantagée» (Gardner, 1995, 16), à côté d'autres groupes dont la position sociale dégradée se manifeste par le fait qu'ils ne bénéficient pas au même titre que les autres des égards dus à chacun dans l'interaction. L'expérience féminine de la ville se caractérise ainsi, d'abord par l'accessibilité excessive à l'interaction (la disponibilité présumée et souvent imposée de façon unilatérale, à l'échange); deuxièmement, par le caractère non sacré de leur intégrité physique, ou "territoriale», dans les termes de Goffman. Gardner compare ainsi les femmes aux enfants qui sont des «open persons» (Goffman, 2013), c'est-à-dire des personnes dont le capital de sacralité (lequel justifie les précautions et la distance cérémonieuse qui marquent le respect pour autrui dans les rapports ordinaires de sociabilité) n'est pas entier.

Gardner définit les pratiques de harcèlement public des femmes comme une somme de conduites disparates néanmoins reliées entre elles par celles auxquelles elles s'adressent - qui forment ainsi le négatif des conduites galantes:

[C'est] cet ensemble d'abus, de tracas, de nuisances et d'irritations qui est caractéristique des espaces publics, et facilité par les conditions spécifiques de la communication en public. Le harcèlement public inclut les pincements, les coups, les remarques criées, la vulgarité, les insultes, les allusions et sous-entendus pernicieux, les œillades appuyées et le fait d'être suivie. Le harcèlement public se trouve le long d'une ligne 
continue d'événements possibles, qui commence au moment où la civilité d'usage entre inconnus est abrogée, et qui finit dans le passage au crime ${ }^{[9]}$ : agression, viol, ou meurtre. (Gardner, 1995, 4)

Le spectre des conduites ainsi désigné est très ample, quoique circonscrit. C'est un ensemble d'actes dont la spécificité tient à la nature publique des situations, à la temporalité des interactions (furtives, anonymes) et à leur portée concrète: des nuisances voire des souffrances, pour celles qui en sont l'objet. L'innovation conceptuelle permet d'objectiver et d'unifier un phénomène jusque-là perçu comme négligeable et anodin, voire considéré comme relevant de l'hommage ou du compliment. Plus précisément, elle permet de constituer une kyrielle de micro-troubles (Emerson, Messinger, 2012) et de situations négligeables, jusqu'alors à peine racontables, en un phénomène identifié de tou·te·s, descriptible, susceptible d'être saisi comme élément d'un tout qui le dépasse et lui donne sens et portée.

Si le débat public français récent a insisté sur les querelles de qualification et les difficultés d'interprétation que pourraient susciter les comportements relevant du harcèlement de rue (au prétexte notamment de confusions possibles avec des formes maladroites de séduction, ou de «drague lourde ${ }^{[10]}$ »), il est intéressant de noter que, quoique pour des raisons entièrement différentes, Gardner inclut dans son analyse, de même que Goffman avant elle, l'ensemble des comportements de séduction et de "parade sexuelle» (Goffman, 2004), y compris non violents et même notoirement bienveillants, tels que ceux qui relèvent de la galanterie: préséances, gestes de protection, etc. ${ }^{[11]}$. L'ensemble des façons d'organiser, y compris dans les rapports de séduction les plus agréables, ce que Gardner appelle «l'accaparement masculin de l'initiative» contribue à rendre possible le phénomène du harcèlement de rue, sans en relever stricto sensu.

Il faut là distinguer deux versants de la réalité, deux dimensions du phénomène et deux niveaux de l'analyse: d'une part, on désigne un

9 Nous soulignons. On trouve ici une première formulation de la continuité temporelle supposée, qui "commence» avec la rupture banale de civilité, et poursuit son évolution jusqu'à "finir» dans "le passage au crime».

10 Voir les débats qui ont fait suite à la tribune sur le "droit d'être importunée», initiée par la romancière Sarah Chiche, et co-signée l'actrice Catherine Deneuve et d'autres personnalités du monde de la culture, parue dans Le Monde le 9 janvier 2018.
11 Les pratiques de galanterie se voient prises dans la critique proposée par Gardner du fait qu'elles entérinent un accaparement masculin de l'initiative, et qu'elles reposent sur un "diagnostic d'incompétence" et de fragilité des femmes, qu'elles contribuent à accomplir et à perpétuer. Sur les implications de cette critique et le trouble normatif qui marque les rapports de genre en public, voir Gayet-Viaud, 2009. 
ensemble de pratiques restreint, qui définit de façon spécifique des situations et des comportements particuliers, qu'il s'agit de décrire et de dénoncer sous le terme de «harcèlement public» des femmes. D'autre part, on articule une diversité de conduites et d'attitudes, prenant place dans une variété d'espaces et de contextes sociaux, qui relève d'une culture partagée des rapports de genre et de hiérarchie des sexes, allant des formes d'égards (ou leur absence) aux formes de violence. Bien sûr, ces deux plans ne sont pas indépendants: ils sont même étroitement reliés par l'analyse. Mais ils ne se confondent pas pour autant: le premier constitue l'arrière-plan normatif (explicatif) du second.

Le harcèlement public des femmes désigne ainsi un ample spectre de conduites, dont le degré de nuisance (voire de violence) varie considérablement, mais qui tient sa cohérence de la culture sexiste dont il est une manifestation.

Documenter un phénomène collectif : la constitution du harcèlement de rue en objet d'étude

La reconnaissance de l'inscription de ces conduites d'apparence anodines, disparates ou sans conséquences, dans un fait social global, relevant du sexisme ordinaire, permet de mettre fin à la négligence ou à l'euphémisation de ces situations, qui jusque-là prévalaient. Elle offre un principe de mise en série de quantité d'épisodes qui pris isolément semblaient négligeables, inoffensifs et presque innocents en un phénomène pénible, pesant, structurant l'expérience féminine de la ville et de ses espaces publics, déterminant une inhospitalité spécifique de ces espaces pour les femmes, a fortiori pour les plus jeunes et lorsqu'elles sont seules (SSMSI, 2018; Lebugle, 2017).

Ainsi décrit pour la première fois comme un phénomène (un fait social), le harcèlement public des femmes montre et dénonce: 1 ) le caractère discriminatoire de ces conduites; 2) le caractère de victimation lié à la dimension cumulative de ces épisodes répétés constitutifs d'une véritable expérience; 3 ) les effets d'inhospitalité des espaces urbains pour les femmes: «le harcèlement public suggère que la place des femmes (le lieu où elles ne sont pas bousculées ni mises en danger) est toujours à la maison» (Gardner, 1995, 12).

D'autres travaux contribuent ensuite à documenter le phénomène. Plusieurs dénominations sont proposées, dessinant des cadrages 
variables: «bystander sexism», "street sexual harassment» (di Leonardo, 1981), "public harassment» (Gardner 1995), "stranger harassment» (Fairchild, Rudman, 2008), "offensive public speech" (Nielsen, 2009). C'est le terme de «harcèlement de rue» qui finit par s'imposer - c'est donc l'expression retenue ici. La plupart des définitions ont insisté sur le fait que ce qui définit la pratique est le fait qu'elle provienne d'hommes et vise des femmes, en public (Bowman, 1993; di Leonardo 1981; MacMillan et al., 2000). Certaines définitions réduisent le spectre des cibles potentielles, comme le remarque Logan (2015) à propos de la définition de di Leonardo: "le harcèlement de rue existe lorsqu'un ou plusieurs hommes inconnus abordent une femme qu'ils perçoivent comme hétérosexuelle dans un lieu public qui n'est pas le lieu de travail de cette ou ces femmes» (di Leonardo, 1981, 51-52). Bowman définit le «harcèlement de rue» comme un «harcèlement sexuel qui est exercé en public sur des femmes par des hommes qui leur sont étrangers" (Bowman, 1993, 519). Mais les cibles ne se limitent pas aux femmes perçues comme hétérosexuelles et en dehors de ce périmètre hétéro-normatif binaire, des populations plus larges sont visées par ces formes de harcèlement, y compris des hommes (Logan, 2015; Pain, 2001; Malbois, 2011).

Les travaux les plus récents se montrent attentifs à la pluralité des victimes de la violence de genre. Kearl définit le harcèlement de rue comme l'ensemble des «interactions malvenues (unwanted) dans les espaces publics entre inconnus (strangers) qui sont motivées par ce qui, à tort ou à raison, est perçu du genre d'une personne, de son orientation sexuelle, ou de son expression de genre, de sorte à produire chez la personne harcelée un sentiment de gêne, de colère, d'humiliation ou de peur $^{[12]}$ » (Kearl, 2010, 5). Mais ces efforts laissent encore de côté l'appartenance ethno-raciale ${ }^{[13]}$ : l'attention aux dimensions intersectionnelles des phénomènes de discrimination (Steinbugler, 2005) permet de considérer la diversité des "axes de harcèlement» tels que la race, la classe, le handicap, la religion, l'âge et l'orientation sexuelle (Fileborn, 2018). Welsh et al. (2006) plaident pour incorporer également le statut citoyen, et prendre en compte l'éventuelle précarité administrative.

En revanche, peu d'études portent sur les auteurs des pratiques de harcèlement de rue. Logan (2015) en répertorie quelques-unes: Benard et Schaffler ont mené une étude dans laquelle la majorité des hommes interrogés partagent l'idée que les comportements relevant du harcèlement de rue sont amusants, inoffensifs, offrent un remède à l'ennui,

12 Notre traduction.

13 Les études indiquent une expérience de victimation des propos offensants publics différenciée en fonction du genre et de la couleur de peau. Voir Nielsen, dans ce numéro. 
donnent un "sentiment de camaraderie joyeuse" (Benard, Schaffler, 1984, 71, cité par Logan, 2015). De la même manière, Wesselman et Kelly (2010) ont établi que la sociabilité masculine est un facteur primordial dans la pratique du harcèlement de rue (Logan, 2015, 204) ${ }^{[14]}$.

La notion a été développée à partir d'études centrées sur les effets que les comportements de harcèlement de rue produisent sur les victimes: ces expériences répétées infléchissent en profondeur l'usage qu'elles font de la ville, portant atteinte à leur liberté de circuler, suscitant des pratiques d'évitement, entretenant chez elles un sentiment de peur, d'insécurité et de vulnérabilité (McDowell, 1999; Fairchild, Rudman, 2008; Stop Street Harassment, 2014; Vera-Gray, 2016, 2018; Condon et al., 2005; Lieber, 2008; SSMI, 2018; Noble, 2015), suggérant qu'elles n'ont pas la même légitimité à se trouver en public que les hommes. Cette illégitimité est diversement mise en scène, et se trouve plus appuyée en certains lieux et à certaines heures du jour et surtout de la nuit, où la simple présence des femmes semble indiquer une disponibilité sexuelle et/ou de mœurs marginales (Kennedy, 2008) - de même que les vêtements qu'elles portent, toujours mis en cause pour entretenir une responsabilisation plus ou moins tacite des femmes victimes d'agression sexuelle ${ }^{[15]}$.

Le concept de harcèlement de rue s'est adossé à l'application aux rapports en public, furtifs et anonymes, d'un concept qui a été central dans l'histoire de la lutte contre les violences faites aux femmes: le concept de harcèlement sexuel. Ce dernier a été d'abord pensé (et continue d'être utilisé prioritairement) pour désigner des formes de violences sexuelles subies par les femmes sur leur lieu de travail (McKinnon, 1979; Logan, 2015). C'est l'exposition répétée aux gestes déplacés provenant d'une même personne, aggravée par l'existence

14 La perspective masculine est présente aussi dans l'analyse du "vandalisme interactionnel" des sans-abri noirs vendeurs de journaux de rue à Greenwich étudié par M. Duneier (Duneier, Molotch, 1999), sans que ces interactions soient thématisées en termes du harcèlement de rue. Ces témoignages rejoignent les quelques récits que nous avons pu recueillir au fil de nos propres enquêtes, où prévalait l'euphémisation des conséquences, le cadrage ludique et la valorisation d'une complicité amicale entre hommes.

15 Ce que traduisent parfois les conseils de prudence diffusés par les institutions publiques de sécurité. Des propos tenus par un responsable de la police canadienne invitant les femmes qui ne veulent pas se faire agresser à ne pas s'habiller de façon provocante ont initié la vague mondiale de protestations des "slut walks", la première d'entre elles s'étant tenue en 2011 à Toronto, puis aux États-Unis, au Canada, en Argentine, en NouvelleZélande et en Europe. Sur ces marches visant à retourner le stigmate, voir Reger (2015). En France plus récemment, une levée de boucliers a été suscitée par la mise en ligne, sur le site du ministère de l'Intérieur, de consignes de prudence adressées aux femmes et faisant porter, là aussi, sur leurs choix de mobilité et leurs choix vestimentaires la responsabilité de s'exposer ou non à des agressions sexuelles (Guenebeaud et al., 2018). 
éventuelle de rapports hiérarchiques plaçant l'auteur de harcèlement en position d'autorité sur sa victime, qui définit le phénomène, et conditionne sa qualification juridique ${ }^{[16]}$. Ainsi, la temporalité s'avère constitutive du préjudice de harcèlement.

L'application du concept de harcèlement au phénomène du harcèlement de rue conduit à passer, dans la définition des actes considérés, d'un auteur unique agissant de façon répétée, dans le cadre d'une relation stable, "ancrée» (Goffman, 1973), dont la victime est captive (pour le harcèlement sexuel sur le lieu de travail par exemple), à une pluralité d'auteurs distincts, non coordonnés ${ }^{[17]}$, et survenant souvent lors d'une occurrence unique, entre personnes qui ne se connaissent pas. Cette opération donne une extension élargie au concept de harcèlement, dont la justification est sous-tendue par la mobilisation d'une seconde notion: celle de «continuum de la violence sexuelle».

\section{Le continuum de la violence sexuelle appliqué au harcèlement de rue}

\section{Définir le continuum de violence}

C'est chez Liz Kelly qu'on trouve la première formulation du «continuum de violence», visant à «décrire l'étendue et la variété de la violence sexuelle dans [la] vie» des femmes (Kelly, 1988, 2012, 2019, 20 ${ }^{[18]}$ ). L'autrice précise que c'est dans «le sens commun du terme continuum» qu'elle utilise le terme:

L'Oxford English Dictionary propose deux définitions en usage: "un caractère commun fondamental qui sous-tend de nombreux événements différents" et "une série continue d'éléments ou d'événements qui se confondent et ne peuvent être distingués d'emblée». La première définition permet de discuter de la violence sexuelle dans son sens général: le principal dénominateur commun à ces événements très divers est que les hommes usent d'une variété de formes d'abus, de

16 Les quatre traits principaux qui encadrent la définition pénale du harcèlement sont: 1) la fréquence 2) I'intensité 3) l'existence d'une relation de pouvoir et 4) la nature même des actes commis et de l'atteinte induite.

17 Ce qui diffère dans le cas des «raids numériques" que la loi récente sur la lutte contre les violences sexuelles vise également, où une pluralité d'auteurs est engagée mais à la suite d'un mot d'ordre ou d'une intention qui les organise comme collectif agissant de façon délibérée et conjointe. Voir Balanant, Rixain, 2017.

18 La traduction française est parue tout récemment dans Delage et al., 2019. 
contraintes et d'usages de la force pour contrôler les femmes. La seconde définition permet de décrire et de nommer cette palette de comportements d'abus, de contraintes et d'usages de la force auxquels sont confrontées les femmes. (Kelly, 2019, 20)

L. Kelly invite toutefois à ne pas confondre l'idée de continuum avec une homogénéisation de l'ensemble et la diversité des comportements violents. Il s'agit plutôt de penser les liens qui existent entre eux, sans les ramener à l'unité:

Un grand nombre de facteurs affectent le sens que prennent pour les femmes les actes de violence sexuelle, et leur impact immédiat et ultérieur. On compte parmi ces facteurs: la nature spécifique de l'agression, la relation existant entre l'homme et la femme ou la fille, le fait qu'il s'agisse d'un évènement isolé ou récurrent, l'ampleur de la menace perçue par la femme au moment et dans le contexte de l'agression, mais également la façon dont elle définit le comportement de l'homme, et si cela renvoie à des expériences antérieures. (Kelly, 2019, 22)

Le concept de continuum est toutefois mobilisé pour questionner la pertinence des distinctions opérées entre types de violences en fonction de leur degré de gravité. Ainsi, évoquant la peur de la mort éprouvée par certaines femmes victimes d'exhibition sexuelle, Liz Kelly insiste sur le fait que «créer une hiérarchie des violences en fonction de leur gravité n'est pas pertinent», et qu'on "pourrait aussi bien avancer (quoique cet argument ne soit pas utilisé ici) que des formes de violence sexuelle, telles que la menace de viol et le harcèlement de rue auxquels la plupart des femmes sont confrontées et qui ont pour effet de limiter leur accès à l'espace public ainsi que leur liberté de mouvement, sont plus graves que, par exemple, la violence domestique, laquelle touche moins de femmes.» (Kelly, 2019, 22)

Finalement, Liz Kelly propose de considérer que «toutes les formes de violence sexuelle sont graves et ont des effets» et que par conséquent "la polarisation ("plus ou moins») du continuum se rapporte uniquement à leur fréquence» (Kelly, 2019, 23). Dans cette perspective, la prévalence d'un ensemble d'actes l'emporte sur les conséquences d'un acte donné, pris isolément. Cela renverse l'échelle ordinaire d'évaluation de la gravité des actes violents. C'est là, pour l'autrice, une façon de prendre en compte le fait que les violences «les plus communes sont aussi les plus susceptibles d'être définies par les hommes comme des comportements 
acceptables $^{[19]}$, par exemple le fait de considérer le harcèlement sexuel comme "un petit jeu» ou "juste une blague», et elles ont moins de chances d'être définies légalement comme des délits.» (Kelly, 2019, 23).

Le succès du concept, point sur lequel Kelly insiste, tient à sa capacité à affirmer «un lien entre toutes les formes de violence sexuelle» (Kelly, 2019, 23), à «mettre en évidence le lien entre le comportement masculin ordinaire et quotidien et [...] les "extrêmes» [...]», et donc à "relier les abus les plus communs et quotidiens [...] avec les épreuves les moins communes qualifiées de délits» (Kelly, 2019, 24-25, 35).

La question qui se pose est, dès lors, celle de la nature de ce lien: entre l'affirmation d'un apparentement, d'une affiliation commune à une culture sexiste, et l'affirmation d'un lien de type causal, l'écart est important.

\section{Dramatiser pour politiser}

et défaire la banalisation

Au fil de sa publicisation, le pro-

blème public du harcèlement de rue transforme l'expérience des femmes et élargit la palette de ressources disponibles pour percevoir, définir et répondre de ce que représentent ces expériences d'interactions subies dans leur vie quotidienne. Fleetwood (2019), qui a étudié 500 récits écrits à la première personne et adressés au site londonien de l'association Hollaback! ${ }^{[20]}$, montre à partir d'une analyse quantitative de contenu menée sur ce corpus qu'il existe désormais une grande «diversité des stratégies utilisées par les femmes, lesquelles incluent le fait de répondre, demander à des tiers leur aide, se défendre physiquement, s'éloigner, et une gamme de "petites» actions quotidiennes et de gestes qui ont pour but de résister au harcèlement» (Fleetwood, 2019, 1). L'autrice avance aussi, à partir de l'idée d'habitus narratif (narrative habitus), que les récits de Hollaback! ne se contentent pas de décrire le harcèlement, mais permettent la constitution de dispositions et de compétences d'auto-défense pour les lectrices de ces témoignages.

19 Kissling (1991) va plus loin dans sa façon de connecter le phénomène du harcèlement à un système sexiste plus large et général, en faisant du harcèlement sexuel l'un des instruments d'un "terrorisme sexuel" qui ser à rappeler aux femmes leur vulnérabilité à l'objectification et aux formes d'agression sexuelle.

20 L'association américaine Hollaback! figure parmi les plus anciennes associations féministes à s'être consacrées spécifiquement à la lutte contre le harcèlement de rue: à partir de 2005 un groupe de militant.e.s documente sur son site internet les expériences de harcèlement vécues par les femmes, pour dénoncer leur violence, leur banalité, et l'indifférence générale dans laquelle elles se produisent. Des initiatives voisines ou directement apparentées (franchises dans des dizaines de pays) naissent dans la décennie suivante. 
L'interaction urbaine quotidienne se découvre ainsi comme un lieu de manifestations des inégalités de genre, mais aussi un lieu possible de leur remise en cause ${ }^{[21]}$. Dans nos propres enquêtes, plusieurs récits de femmes soulignent le devenir militant et pédagogique de certaines de ces situations (Bidet et al., 2020) : plusieurs femmes racontent devenir, avec le temps, plus enclines à répondre, à réagir, voire à revenir sur leurs pas pour sermonner, parfois avec succès, ceux qui les sifflent ou leur font des remarques désagréables et sexistes sur leur passage. Les entretiens suggèrent que ces changements d'attitudes se nourrissent de la politisation en cours du sujet: la possibilité et la nécessité de réagir infusent au fil des témoignages et analyses lus et entendus sur le sujet, des discussions amicales, de l'appropriation par les femmes du terme de «harcèlement de rue» et de sa portée dénonciatrice, toutes choses qui concourent à considérer comme déplacées et inacceptables les conduites ainsi désignées. Ces transformations semblent aussi se faire, pour certaines, à la faveur de la découverte par leurs propres filles de cette expérience pénible, à la puberté.

Les bénéfices de la publicisation du harcèlement de rue peuvent donc être pensés à deux niveaux. Au niveau individuel d'une part, la constitution d'un problème public donne une intelligibilité à une série d'épisodes inscrivant les expériences personnelles dans l'expérience collective, justifiant la charge émotionnelle souvent conçue jusque-là comme irrationnelle ou disproportionnée ${ }^{[22]}$. Le premier bénéfice concerne donc les femmes victimes, à l'échelle individuelle: il leur permet de s'inscrire dans une expérience commune, collective, de faire émerger un sujet collectif; de qualifier l'expérience vécue comme violence subie. Le second bénéfice relève de l'opération de politisation rendue possible: la désignation de ces actes comme ensemble cohérent donne prise à l'action publique, en inscrivant des comportements jusque-là vus comme anecdotiques et anodins dans le périmètre d'un phénomène global aux conséquences importantes et dignes de mobiliser l'action publique, ce qui rétroagit sur le niveau individuel, et transforme la définition de ce qui était banal, normal, et devait simplement être supporté, en quelque chose qui n'est ni normal ni tolérable, et qu'on ne peut donc pas laisser passer ${ }^{[23]}$.

La catégorie ne permet pas, en revanche, de résoudre entièrement le problème posé par le caractère dissymétrique de l'expérience ainsi

21 Sur cette double valence des interactions en public et de la normativité qui y est mise à l'œuvre, voir Gayet-Viaud et al., 2019.

22 Sur cette tendance à considérer les événements de la sociabilité publique urbaine comme quantités négligeables, voir GayetViaud, 2011
23 Cela a nourri le développement de formes de solidarité entre femmes en public, face à ces situations. L'association Stop Harcèlement de rue a d'ailleurs fait de l'intervention des témoins l'un de ses axes de communication (voir la contribution de M. Dekker dans ce numéro). 
reconfigurée. Demeure quelque chose qui, dans la situation, relève de l'écart irrémédiable des points de vue: là où chaque situation représente une occurrence isolée pour les auteurs de commentaires, elle s'inscrit dans une série qui en articule des dizaines voire des centaines, pour les récipiendaires. L'opération de qualification en «harcèlement» renverse, de ce point de vue, la situation précédente: l'euphémisation favorisait la seule perspective masculine, et négligeait le point de vue des victimes. La dramatisation tend au contraire à homogénéiser le groupe des auteurs, au titre des conséquences collectives de leurs comportements. En inscrivant chaque auteur dans ce périmètre redéfini au niveau collectif, la notion l'inscrit de fait dans un phénomène dont les conséquences doivent leur importer même si elles sont, possiblement, fort déconnectées de ses intentions individuelles, ou de la perception de ses actes propres.

Cette requalification de la conduite individuelle au titre de ses conséquences pour la vie commune, qui est une manière de définir la politisation d'un problème, semble aussi importante que féconde: elle contribue à transformer la normativité des rapports de genre en public. Pour autant, ce processus de reconfiguration et de traduction politique du phénomène, qui s'est, on l'a vu, adossé à l'idée de continuum, a emporté une autre forme de requalification plus discutable: le phénomène désigné, qui portait sur une série d'actes distincts relevant tous de la violence sexiste, tend désormais à être pensé à travers le prisme d'une dynamique de gradation, se déployant au niveau même des situations et des auteurs, considérés individuellement.

En effet, la pénalisation en cours s'adosse, comme on va le voir, à l'existence présumée d'une logique d'engrenage. La compréhension politique du phénomène va, de ce fait, jouer de l'ambiguïté charriée par l'idée de continuum. De la parenté conceptuelle, on va passer subrepticement à la causalité (ou du moins, au déploiement chronologique probable). Autrement dit, on va rétroactivement appliquer aux occurrences singulières et aux individus les motifs identifiés via la considération des conséquences collectives.

Ce glissement, qui tient notamment à un usage contestable du concept de continuum, présente des analogies notables avec les logiques qui ont été mobilisées pour penser et porter les politiques de lutte contre l'incivilité. Celles-ci se sont, en effet, appuyées elles aussi sur le concept de continuum de violence, mobilisant des logiques rhétoriques voisines. Elles ont ainsi conduit à consacrer les théories de l'incivilité qui ont prospéré aux États-Unis d'abord, puis en Europe à partir des années 1990. 


\section{Le continuum de violence: aux sources de politiques pénales favorisant une répression "précoce»}

On propose de faire retour sur un autre problème public qui a, dans les décennies récentes, mobilisé le concept de continuum de violence et, ce faisant, contribué à connecter des pratiques inscrites dans les formes routinières de la sociabilité avec des formes lourdes de déviance et de criminalité: le problème public de l'incivilité (Gayet-Viaud, 2019). En revenant sur les conditions d'émergence et de diffusion en France des théories qui ont promu ce concept et porté son succès, on espère apporter un éclairage intéressant à la présente réflexion, et à la compréhension des enjeux politiques dont les théories de «l'engrenage» et leurs usages en matière pénale, sont porteurs.

Le continuum des violences, au

cœur de la politisation

des incivilités

La notion d'incivilité, dans son acception actuelle, a été introduite en France dans les années 1990. Elle a été importée des États-Unis où, désignée surtout comme "disorder» ou "antisocial behavior", elle a tenu une place centrale dans les politiques urbaines de sécurité qui ont été mises en œuvre en lien avec la "Broken Window theory ${ }^{[24]}$ ", laquelle lui attribuait une importance stratégique et inédite. L'idée phare en est que les désordres non sanctionnés nourrissent une "spirale du déclin", par un effet de contagion et d'accumulation des désordres sur un territoire donné, doublées d'une aggravation graduelle des infractions commises, allant de la simple négligence au crime violent. Cet engrenage conduit alors de ce qui n'est initialement qu'une fenêtre brisée dans les parties communes d'un immeuble, à la survenue d'un homicide.

La théorie insiste, de façon tout à fait opportune, sur l'importance des conditions matérielles et situationnelles dans la survenue de comportements déviants, et met l'accent sur le rôle du contrôle social dans la régulation des conduites; mais ce qui en constitue la marque de fabrique la plus nette, et qui surtout détermine le devenir des politiques

24 Voir Kelling, Wilson 1982; Skogan, 1990. Pour une présentation détaillée des conditions de cette importation transatlantique, voir Gayet-Viaud, 2014. Sur les effets de cette configuration des troubles de la sociabilité urbaine en termes d'insécurité et de délinquance, voir Gayet-Viaud, 2019. 
qu'elle a inspirées, c'est la logique qu'elle dessine, en vertu de laquelle il est nécessaire d'éradiquer de façon précoce tous les désordres visibles (vandalisme, incivilités, délits mineurs).

Cette dimension temporelle souligne deux dimensions distinctes de l'engrenage en jeu: la première tient à la concentration et à l'accumulation graduelle de problèmes dans un même territoire; la seconde correspond à l'aggravation progressive des pratiques de déviance. Les politiques de répression qui se sont développées en s'adossant à ces perspectives ont donc considéré la nécessité de couper ces problèmes à la racine, en punissant et en excluant les fauteurs de troubles (Harcourt, 2006) dès les premières phases, les stades précoces, soit avant que ces désordres mineurs ne se multiplient, mais aussi avant que ces déviances, mineures d'abord, ne se développent et ne s'aggravent.

Au cœur de cette théorie et de la justification théorique de cette répression "précoce», se trouve la considération des dynamiques d'engrenage qui relient les comportements les plus anodins, dispersés, apparemment négligeables, à des comportements graves et criminels. Le rôle de la sanction est décisif dans la perspective adoptée: car l'impunité est réputée nourrir ce cercle, en laissant prospérer les comportements non conformes aux règles d'un côté, alors que la sanction se voit attribuer la capacité de couper court à cette pente délétère et à ces élans, supposés manifester un rapport réfractaire aux règles du vivre-ensemble (Roché, 2002).

Le postulat selon lequel les incivilités seraient les prémisses, et donc les signes précurseurs, de violences plus graves, est ce qui a valu à celles-ci de trouver une place de choix dans les analyses policières des émeutes urbaines du début des années $1990^{[25]}$, puis dans le développement de politiques locales de sécurité au fil des décennies suivantes. Or, l'unité du phénomène n'allait pas de soi, puisqu'il désignait de façon assez vague une fourchette ample et hétérogène de conduites, comme cela a été souligné maintes fois. Deux dimensions ont été mises en avant pour justifier leur regroupement: d'une part, le rapport de mépris qu'elles étaient réputées manifester vis-à-vis de la chose commune; d'autre part, leur caractère infra-légal, et l'absence de qualification juridique décrite comme source du problème et de l'impossibilité de sanction. La possibilité de sanctions pénales a ainsi été considérée comme la réponse obligée au phénomène. 
La pénalisation a ainsi été promue comme la seule façon possible d'endiguer des comportements d'incivilité, dont l'essor était vu comme proportionnel à l'impunité dans laquelle ils prospéraient. Leur appréhension depuis la perspective de ce qu'ils pourraient engendrer, et l'horizon des crimes auxquels ils sont présumés pouvoir conduire si on ne les stoppe pas de façon «précoce», «en amont»-est ce qui a porté les politiques, désormais célèbres, dites de tolérance zéro (Harcourt, 2006). Cette politisation a ainsi justifié une production législative abondante, au cours de la décennie 2000 notamment, entraînant la pénalisation ou l'aggravation des sanctions visant les désordres publics et, par glissement, la focalisation de l'effort répressif sur certaines pratiques et populations: les mendiants, les prostitués, les jeunes occupant des halls d'immeuble, les vendeurs à la sauvette.

L'idée qu'il n'existe qu'une différence de degré et non de nature entre les ruptures mineures de la civilité et les formes de violence les plus graves a conduit à considérer la nécessité de réprimer les premières en proportion de la gravité de ce à quoi elles peuvent mener. La perception des premières a ainsi été définie depuis la possibilité de leur développement dans les secondes.

Une pénalisation articulée

à l'anticipation des

aggravations futures

Le parallèle est frappant si l'on considère la définition officielle du harcèlement de rue récemment adoptée par les pouvoirs publics. Celle-ci pose, certes, le caractère en soi répréhensible des formes de harcèlement de rue, mais l'accent est cependant rapidement placé, dans la justification de l'intervention publique, sur leur possible aggravation dans des «formes d'agression plus graves»:

Harcèlements sexistes et violences sexuelles se manifestent de différentes manières: des sifflements, des regards appuyés, des jeux de langues, des actes sexuels mimés, des frottements, des pincements de fesses, des caresses, etc. Les premières manifestations du harcèlement telles que les sifflements, les commentaires sur la tenue vestimentaire ou le physique, sont encore trop souvent banalisées, tolérées. Ces formes de harcèlement sont inacceptables en soi. De plus, si elles ne sont pas stoppées, elles peuvent parfois mener à des formes d'agression plus graves telles que des injures, des 
menaces ou des agressions sexuelles tombant sous le coup de la loi. (Site du Gouvernement «Réagir contre le harcèlement sexiste et les violences sexuelles "[26])

Dans son intervention devant la commission sénatoriale siégeant pour préparer la loi contre l'outrage sexiste, la secrétaire d'État Marlène Schiappa formule cette thèse de façon plus directe encore:

Nous travaillons également, sous la houlette du ministère de l'Intérieur, sur ce que nous avons appelé les quartiers de reconquête républicaine où 10000 policiers de la sécurité du quotidien verbaliseront, notamment, le harcèlement de rue. [...] Ma conviction, celle des experts, est qu'il existe un véritable continuum des violences, depuis le harcèlement de rue jusqu'au viol: ces comportements ne sont pas décorrélés, la plupart des violeurs avaient en effet déjà agressé sexuellement ou déjà harcelé. Tous les policiers que nous avons auditionnés nous expliquent que les violences sexuelles suivent une forme de gradation exponentielle. [...] je n'avance pas que quelqu'un qui harcèle dans la rue va devenir systématiquement un violeur mais je soutiens qu'il faut bloquer le processus immédiatement et pour cela ne rien laisser passer, même la plus "petite» des agressions ou des atteintes sexuelles à l'encontre des femmes. (Balanant, Rixain, 2017, 62)

Cet extrait appelle deux remarques. D'une part, on voit que le cadrage topographique est considéré comme une évidence: c'est dans «les quartiers de reconquête républicaine» que l'on juge utile de focaliser l'investissement répressif, en termes de présence policière et de verbalisation des outrages. D'autre part, la secrétaire d'État explicite sa vision de la notion de continuum des violences: le lien ici affirmé entre le harcèlement de rue et le viol n'est pas un lien analytique décrivant une "culture du viol». Il ne vise pas un arrière-plan culturel commun, mais désigne une continuité empirique, chronologique, voire causale, chez les auteurs mêmes.

Surtout, ce lien est décrit comme opératoire à un niveau qui n'est plus seulement collectif (via l'idée que le sexisme se nourrit de pratiques jugées bénignes et euphémisées qui contribuent à rendre possibles des

26 https://www.egalite-femmes-hommes.gouv.fr/dossiers/lutte-contre-les-violences/ harcelement-transports/de-quoi-parle-t-on/. 
actes plus graves) mais individuel: le processus s'applique à l'évolution d'une seule et même personne, dont la pente (glissante) doit être «bloquée» dès que possible. Malgré une prudence relative, puisque la secrétaire d'État se défend d'endosser une vision causaliste, c'est bien un rapport de cause à effet qui est suggéré: «la plupart des violeurs avaient déjà agressé sexuellement ou déjà harcelé». La stratégie politique mise en œuvre s'ordonne donc à l'existence de cette "gradation exponentielle», peu documentée, qui conduit, si ce n'est pour tous les harceleurs, du moins pour une partie, semble-t-on devoir comprendre, du harcèlement au viol. Référence est alors faite aux expériences policières. Or, dans nos propres entretiens auprès d'agents et de cadres de la sécurité publique, les propos recueillis tendent à fortement distinguer la clientèle des "frotteurs» et des agresseurs sexuels de la population générale, et du nombre considérable des auteurs de commentaires sexistes ordinaires, dans la rue et ailleurs ${ }^{[27]}$.

C'est surtout le passage de la reconnaissance d'effets produits à un niveau collectif à l'imputation d'une dynamique à l'œuvre pour chaque individu qui pose problème. L'enjeu est un glissement et une confusion. Pourtant, l'idée que des violences mineures peuvent graduellement conduire à des violences de plus en plus graves n'est pas sans fondement: elle est issue de l'analyse des dynamiques de violences prenant place entre familiers. Le concept de harcèlement a d'ailleurs été pensé, comme on l'a rappelé, pour qualifier des situations qui se répètent avec un même homme violent. Dans le contexte des violences conjugales, par exemple, chaque forme de violence perpétrée représente un seuil et un verrou levé, qui permet qu'un degré supérieur soit franchi la fois suivante. La situation est celle d'une stricte continuité temporelle et relationnelle entre des protagonistes constants, dans un espace lui-même identique, et suivant des logiques relationnelles relevant elles aussi de la répétition.

L'application de cette perspective à une série d'hommes distincts, où la répétition et l'accumulation n'existent que du côté des femmes, ne va pas de soi. Le risque est de voir mise en œuvre une logique du «qui vole un œuf vole un bœuf» (ou volera bientôt un bœuf) qui promeut comme une évidence l'orientation politique visant une répression "précoce», ajustée à la perspective du pire, soit potentiellement peu ajustée en regard des caractéristiques propres des offenses mineures.

27 Les enquêtes de victimation (Hamel et al., 2016) ont documenté la fréquence des formes ordinaires d'offense sexiste en public (leur prévalence frôle les $100 \%$ de femmes interrogées), cependant que les viols sont d'abord le fait de proches et non d'inconnus croisés en ville. 
Les politiques de lutte contre

le harcèlement. Subordonner

la considération des actes mineurs

aux actes les plus graves

Les campagnes de communication conduites en France ces dernières années dans le cadre des politiques de prévention donnent à voir quelques traductions politiques possibles des cadrages conceptuels que l'on vient d'évoquer: la rhétorique de la dénonciation du harcèlement de rue développée prend pour pivot le geste d'agression sexuelle. On peut prendre pour exemple la campagne de communication pour la prévention du harcèlement dans les transports publics, menée au printemps 2018 par voie d'affichage dans les stations de métro de la RATP, qui est à cet égard exemplaire.

Sur chacune des affiches, l'avertissement «Ne minimisons jamais le harcèlement sexuel" accompagne une mise en scène dramatique qui montre une jeune femme, telle une proie, s'agrippant à la barre de maintien du métro, crispée, menacée par un animal sauvage (un ours, un requin ou une meute de loups), lesquels se trouvent juste derrière elle, transformant l'environnement (la rame de métro) en grotte (ou tout autre milieu sauvage et hostile, sous-marin ou forestier) la victime y étant isolée (nulle âme secourable en vue), crispée, passive et visiblement terrifiée. Cette présentation incarne la paradoxale reconnaissance accordée aux offenses dont les femmes sont victimes quotidiennement: pour en affirmer l'existence et l'importance, la gravité propre est négligée, au profit d'une affiliation à des actes plus graves, qui conditionnent la prise au sérieux des comportements désignés.

Au moment d'affirmer l'illégitimité des gestes d'irrespect et de sexisme ordinaire banalisés dans la vie quotidienne, on convoque des formes de violence autres (plus graves), pour inciter à prendre les premières au sérieux. Pour affirmer leur importance, on en vient paradoxalement, à les rapprocher de - voire à les assimiler avec-des actes et situations distincts qui désignent, sur le plan des atteintes à l'intégrité physique comme de la dangerosité perçue, des seuils de gravité nettement supérieurs: en particulier l'agression sexuelle et l'attouchement. Le message devient: l'offense mineure est grave, parce qu'elle peut mener à des formes de prédation d'une violence bien supérieure...

Ces rhétoriques de la dramatisation présentent plusieurs points communs avec les logiques qui se sont mises en place au fil des reprises politiques et médiatiques de l'incivilité, en caricaturant la «sauvagerie» supposée des auteurs d'incivilités. Elles contribuent ce faisant à diffuser des perceptions discutables, voire potentiellement contre-productives

Le harcèlement de rue et la thèse du continuum des violence 
des problèmes, notamment pour la définition des réponses publiques pertinentes à apporter. La pertinence du cadrage du problème dans les termes d'une dangerosité supposée partagée entre auteurs (d'offenses sexistes, d'agressions ou de crimes) reste un point aveugle de ces rhétoriques préventives. La thématisation d'une menace physique et d'une dangerosité est-elle incontournable pour promouvoir une civilité égalitaire dans les rapports hommes-femmes en public? Est-il nécessaire et opportun de considérer que c'est au seul titre de son rôle de prémisse de la violence physique que la réification des femmes et leur sexualisation imposées en public peuvent/doivent être combattues?

\section{Conclusion. Perceptions des faits et des situations dans les politiques publiques de réponse aux pratiques de harcèlement de rue}

Pendant trois décennies, malgré un cadrage théorique qui aurait amplement justifié que les offenses sexistes (et plus généralement les violences verbales) soient prises en compte et en charge dans les politiques urbaines de lutte contre les incivilités, les offenses sexistes (de même que d'autres désordres urbains, également peu visibles et négligés du fait de leur absence de traces matérielles ou de coût économique direct ${ }^{[28]}$ ) ont été négligées, passées sous silence et laissées entièrement de côté (littéralement hors champ) par les politiques de régulation des espaces publics urbains ${ }^{[29]}$. Les incivilités ont donné lieu à des politiques hautement discrétionnaires, se focalisant sur des territoires, des problèmes et des publics très sélectifs. Il est à craindre qu'il en soit de même pour l'outrage sexiste ${ }^{[30]}$.

Or, les conditions d'applicabilité de la loi ${ }^{[31]}$ constituent bien évidemment un aspect important du devenir pénal du problème public du harcèlement de rue. Les travaux préparatoires à la rédaction et au vote de la loi ont d'ailleurs eu à cœur de s'assurer de son applicabilité et de ne pas en faire une "toothless law», au fond impuissante (Fielborn,

28 Sur les définitions très variables de l'incivilité par les divers acteurs de sa prise en charge, et leurs conséquences sur les politiques conduites, voir Gayet-Viaud, 2014 et 2017.

29 Les enquêtes (telles que l'enquête "Cadre de vie et sécurité») montrent que l'insulte est la forme la plus fréquente de l'offense publique sexiste (Lebugle, 2018).
30 Voir la contribution de Marylène Lieber dans ce même numéro.

31 Sur la place de cette inquiétude dans les réticences vis-à-vis de la pénalisation, voir aussi la traduction du texte classique de L. B. Nielsen dans le présent numéro. 
Vera-Gray, 2017) ${ }^{[32]}$ ». Mais la plausibilité de l'offense risque bien d'être, en pratique, étroitement corrélée aux conditions perçues de la dangerosité des auteurs: d'autant plus que c'est cette dimension qui désormais structure la considération politique du problème. L'ensemble des conditions pratiques (menace perçue, disponibilité d'une intervention répressive) risque dès lors de produire une répression discrétionnaire et donc très inégalement distribuée en fonction des territoires et des populations.

S'agissant des auteurs d'abord: là comme en d'autres aspects du traitement de l'incivilité, le risque est fort que la lutte contre le harcèlement de rue, dont les justifications se sont adossées à une compréhension très ample du phénomène des violences de genre, ne cible pour finir, en aval, que les formes les plus visibles et les auteurs les plus accessibles à l'intervention policière - en l'occurrence, une population qui passe un temps important dans la rue et les espaces publics urbains, où se déroulent une part importante de sa sociabilité et de ses activités économiques. Le déséquilibre pourrait nourrir et contribuer à produire une délinquance de rue qui est d'autant mieux connue qu'elle est préférentiellement visée ${ }^{[33]}$, comme le suggère la mention explicite par $M$. Schiappa des «quartiers de reconquête républicaine».

Des rétrécissements du champ d'attention également problématiques sont à craindre du côté du périmètre des victimes reconnues comme telles. Certaines femmes (des prostituées ${ }^{[34]}$ aux femmes voilées ${ }^{[35]}$, qui ont en commun d'être jugées à partir de leur non-conformité à une norme, de décence, ou d'émancipation supposée) sont difficilement considérées comme des usagères de la ville pleinement légitimes. Ainsi, de même que les offenses subies par diverses minorités (en raison du genre, de l'orientation sexuelle, de la religion, de la race, de l'âge, de la classe ou du handicap) sont restées hors du champ des préoccupations et des politiques de lutte contre les incivilités, qui se sont préférentiellement focalisées sur les désordres et dommages subis par les institutions (celles provoquant des coûts humains et matériels aisément

32 Le site internet www.egalite-femmeshommes.gouv.fr précise: "C'est le cas d'une injure prononcée en pleine rue, sur un quai de métro ou dans un bus. La peine encourue pour une injure publique à caractère sexiste est de 6 mois de prison et de 22500 euros d'amende. L'injure publique est un délit qui relève du tribunal correctionnel. La victime d'injure publique à caractère sexiste peut déposer plainte dans un délai d'un an."

33 Comme l'ont montré les travaux sur la délinquance en col blanc (Katz, 2013
Lascoumes, Nagels, 2014), il est à la fois aisé et politiquement rentable de combattre la délinquance de rue, cependant qu'il est malaisé et politiquement délicat de lutter pour mettre au jour la délinquance en col blanc.

34 Sur d'autres versants des politiques pénales, il a été montré combien les conditions pour être jugée une victime crédible sont exigeantes, et défavorables à certains profils de femmes. Voir Darley, 2019.

35 Voir Chakraborti, Zempi, 2012; Abdmolaei, Hoodfar 2018. 
quantifiables), on peut craindre que le spectre des pratiques de harcèlement susceptibles d'être constatées et verbalisées se trouve réduit en regard des périmètres que les concepts permettaient initialement de dessiner. Il s'agirait, pour prendre au sérieux le problème général de l'offense publique, de lutter aussi contre le sentiment d'insécurité des minorités et de l'ensemble de la population, et contre le sentiment d'impunité (et d'impuissance) qui domine aujourd'hui, dans toutes les situations d'injure discriminatoire.

Le parallèle dressé ici, trop rapidement, avec la conception du phénomène des incivilités et avec les politiques de lutte qui les ont visées, permet d'envisager une dernière question, relative aux modalités de la réponse publique qui peuvent être apportées au problème du harcèlement de rue. Comme on l'a vu, la politisation est allée de pair avec une forme de dramatisation, qui s'est elle-même nouée, via l'idée d'un possible continuum, désigné de façon discutable comme un engrenage, autour de la nécessité affirmée de réprimer les conduites de harcèlement. Les réserves soulevées, du risque d'application discrétionnaire à celui de la stigmatisation, doivent-elles vouer à l'impuissance ou à l'inaction? Loin de là. Ce sont, bien plutôt, les termes mêmes de cette alternative entre une politisation menant fatalement à des politiques répressives et la négligence ou l'affirmation d'une impuissance face au problème qu'il s'agit de questionner. On peut sans doute suggérer, là aussi, quelques enseignements issus des luttes contre l'incivilité.

Le recul offert par celles-ci montre en effet l'importance de formes de présence humaine polyvalente (pas nécessairement policière donc), et plus généralement de l'existence de «prises» données aux usagers et au public, pour lui rendre possible le respect des règles et aussi attrayante que possible la mise en œuvre des égards: ces prises relèvent aussi bien de la qualité de l'environnement matériel, et de son entretien, que de la disponibilité de figures responsables des règles d'usage, chargées de les rappeler le cas échéant, ou offrant des recours en cas de difficulté. Il est alors question d'une compréhension élargie de la prévention, certes coûteuse en ressources humaines (et donc économiques) mais qui n'est pas tant une dépense qu'un investissement, au service de la viabilité d'espaces publics hospitaliers à tous. On continue de sous-estimer le rôle déterminant du retrait progressif, depuis les années 1980, de ces figures de "gardiens des lieux» (Roché, 2000) - des concierges, aux contrôleurs et aux îlotiers - dans la dégradation des rapports sociaux et dans l'essor des conflits entre usagers, mais aussi entre publics et institutions, trop souvent décrits de façon incomplète comme «incivilités» ${ }^{[36]}$. 
De même, ce qui a souvent été décrit comme une apathie croissante des citoyens devant ce qui se passe en public (imputé à l'individualisme rampant, etc.) tient possiblement aux difficultés croissantes qu'éprouve chacun au moment de trouver en cas de difficulté, des interlocuteurs accessibles, disponibles et responsables de ce qui advient, que ce soit dans les transports ou dans la rue. Cette possibilité d'offrir des recours situationnels pratiques, en deçà même de tout recours au droit, donnerait assurément des étais précieux aux réactions de défense et de solidarité que la publicisation du problème a contribué à faire éclore ${ }^{[37]}$. L'alternative entre répression et euphémisation est donc, insistons-y, bien loin d'épuiser le spectre des réponses possibles à apporter.

\author{
Carole Gayet-Viaud \\ CNRS-CESDIP (UVSQ-UCP-ministère de la \\ Justice) et EHESS-CEMS \\ £CESDIP \\ 43, boulevard Vauban, 78280 Guyancourt \\ carole.gayetviaud@cesdip.fr
}

37 Permettre que des prises soient données et des recours accessibles et efficaces soient offerts aux victimes de violences verbales et d'injures discriminatoires subies de la part des agents de police, compléterait heureusement les mesures prises en ce sens. 
ABDMOLAEI S., HOODFAR H., 2018. Porter son identité à l'ère de la mondialisation: politiques du voile et de la mode en contextes musulman et occidental, Anthropologie et Sociétés, 42, 1, 81-111.

BALANANT E., RIXAIN M.-P., 2017, Rapport de la Délégation aux droits des femmes et à l'égalité des chances entre les hommes et les femmes, sur le projet de loi renforçant la lutte contre les violences sexuelles et sexistes, Annexe 1, Compte rendu de l'audition de Marlène Schiappa mardi 17 avril, Assemblée Nationale, Rapport d'information $n^{\circ} 895$, 53-71.

BASTIAT B., 2004, Le mouvement des femmes et l'accès à l'espace public des années 1970 aux années 1990: exemples français, suisses et irlandais, in DENĖFLE S. (dir.), Femmes et villes, Tours, Presses Universitaires François Rabelais.

BATHELIER V., BLIDON M., MATTEI M.F., VALLET B. (dir.), 2017, Annales de la recherche urbaine, "Le genre urbain", 112.

BENARD C., SCHLAFFER E., 1984, The Man in the Street: Why He Harasses, in JAGGER A.M., ROTHENBERG P.S. (Eds.) Feminist Frameworks: Alternative Theoretical Accounts of the Relations Between Women and Men, Third Edition, New York: McGraw Hill, 70-72.

BLIDON M., 2016, Espace urbain, in RENNES J. (dir.), Encyclopédie critique du genre, Paris, La Découverte, 242-251.

BOWMAN C.G., 1993, Street harassment and the informal ghettoization of women, Harvard Law Review, 106, 517-580.

CEFAÏ D., 2012, Présentation, in CEFAÏ D., PERREAU L. (dir.), Goffman et l'ordre de I'interaction, PUF/CURAPP, 6-30.

CENTRE HUBERTINE AUCLERT, 2018, Violences faites aux femmes dans les espaces publics en Île-de-France.

CHAKRABORTI N., ZEMPI I., 2012, The Veil Under Attack: Gendered Dimensions of Islamophobic Victimization, International Review of Victimology 18, 3, 269-284.
CONDON S., LIEBER M., MAILLOCHON I., 2005, Insécurité dans les espaces publics: comprendre les peurs féminines, Revue française de sociologie, 46, 2, 265294.

COUTRAS J., 1996, Crise urbaine et espaces sexués, Paris, Armand Colin et Masson

DARLEY M., 2019, Le proxénétisme en procès et la réaffirmation d'un ordre sexuel national, in GIRARD G., SALLÉE N., PERREAULT I. (dir.) Sexualité, savoirs et pouvoirs, Presses universitaires de Montréal, 155-165.

DELAGE P., LIEBER M., CHETCHUTIOSOROVITZ N. (dir.), 2019, Violences de genre: retours sur un problème féministe, Cahiers du genre, 1, 66.

DENÈFLE S. (dir.), 2004, Femmes et villes, Tours, Presses Universitaires FrançoisRabelais.

DENÈFLE S., RATOUIS O., 2010, Le genre et la ville. Entretien avec Sylvette Denèfle, Métropolitiques, [en ligne] https://www. metropolitiques.eu/Le-genre-et-la-ville. html.

DI LEONARDO M., 1981, Political economy of street harassment, $A E G / S$ : Magazine on Ending the Violence Against Women, 51.

DUNEIER M., MOLOTCH H., 1999, Talking City Trouble: Interactional Vandalism, Social Inequality, and the Urban Interaction Problem, American Journal of Sociology, 104, 5, 1263-1295.

EMERSON R., MESSINGER S., Micropolitiques du trouble, in CEFAÏ D., TERZI C. (dir.) L'expérience des problèmes publics, Raisons pratiques, Éditions de I'EHESS, 57-80.

FAIRCHILD K., RUDMAN L.A. 2008, Everyday Stranger Harassment and Women's Objectification, Social Justice Research 21, 3, 338-357.

FILEBORN B., 2018, Naming the Unspeakable Harm of Street Harassment: A Survey-Based Examination of Disclosure Practices, Violence Against Women 25, 2, 223-248. 
FILEBORN B., VERA-GRAY F., 2017, "I Want to be Able to Walk the Street Without Fear": Transforming Justice for Street Harassment, Feminist Legal Studies, 25, 2, 203-227.

FLEETWOOD E., 2019, Everyday selfdefence: Hollaback narratives, habitus and resisting street harassment, British Journal of sociology, 70, 5, 1709-1729.

GARDNER C.B., 1980, Passing By: Street remarks, Address rights, and Urban Woman, Sociological Inquiry, 50, 3-4, 328-356.

GARDNER C.B., 1989, Analyzing Gender in Public Places: Rethinking Goffman's Vision of Everyday life, The American Sociologist, Spring, 42-56.

GARDNER C.B., 1995, Passing by. Gender and public harassment, University of California Press.

GAYET-VIAUD C., 2009, La femme autonome et l'homme galant. Intransigeances du respect et péripéties de l'égard, in JOUAN M., LAUGIER S. (dir.) Penser l'autonomie, Paris, PUF, 389-406.

GAYET-VIAUD C., 2011, La moindre des choses. Enquête sur la civilité urbaine et ses péripéties, in BERGER M., CEFAÏ D., GAYET-VIAUD C. (dir.), 2011, Du civil au politique. Ethnographies du vivreensemble, Bruxelles, PIE Peter Lang, 2754.

GAYET-VIAUD C., 2014, Le lien civil en crise?, Bruxelles, Yapaka.

GAYET-VIAUD C., 2017, French Cities' Struggle Against Incivilities: From Theory to Practices in Regulating Urban Public Space, European Journal of Criminal Policy and Research, 23, 1, 77-97.

GAYET-VIAUD C. 2019, "La civilité estelle réac?", Politix. Revue des sciences sociales du politique, "Le politique au coin de la rue», 1, 125, 31-58

GAYET-VIAUD C., BIDET A., LE MENER E., 2019, Enquêter sur la portée politique des rapports en public, Politix. Revue des sciences sociales du politique, 1, 125, 7-30.
GOFFMAN E., 2004 [1976], Le déploiement du genre, Terrain, 42, 109-128.

GOFFMAN E., 2013 [1963], Comment se conduire dans les lieux publics. Notes sur I'organisation sociale des rassemblements, Paris, Economica, coll. "Études sociologiques".

\section{GUENEBEAUD C., LE MAT A.,} VERHAEGHE S., 2018, Take back the night! Une exposition pour combattre les violences sexistes dans l'espace public, Métropolitiques, [en ligne] https://www. metropolitiques.eu/Take-back-the-nightUne-exposition-pour-combattre-lesviolences-sexistes-dans-I.html.

GUSFIELD J., 2009 [1981], La culture des problèmes publics. L'alcool au volant: la production d'un ordre symbolique, Economica, coll. "Études sociologiques".

HAMEL C., DEBAUCHE A., BROWN E. (dir.), 2016, Viols et agressions sexuelles en France: premiers résultats de l'enquête VIRAGE, Population et sociétés, 538.

HARCOURT B., 2006 [2001], L'illusion de I'ordre: Incivilités et violences urbaines: tolérance zéro? Paris, Descartes et Cie, coll. "Les urbanités".

HCE/HF, 2015 Avis sur le harcèlement sexiste et les violences sexuelles dans les transports en commun, [en ligne] https:// haut-conseil-egalite.gouv.fr/IMG/pdf/ hcefh_avis_harcelement_2015-04-16vio-16.pdf.

IAU, 2016, Enquête victimation et insécurité: les spécificités de genre, Note rapide $n^{\circ} 722$

KATZ J., 2013, L'ethnographie en trois dimensions, Laviedesidées, https:// laviedesidees.fr/L-ethnographie-en-troisdimensions.html.

KEARL H., 2010, Stop street harassment: Making public places safe and welcoming for women. ABC-CLIO.

KELLING G. L., WILSON J. Q., 1982, Broken Windows, Atlantic Monthly, 249, 3, 29-36, 38. [Trad. Fr.: 1994, "Les vitres cassées", Les Cahiers de la sécurité intérieure, 15, 163-180]. 
KELLY L., 1988, Surviving sexual violence, Oxford, Polity Press.

KELLY L., 2012, Preface, in BROWN J.M., WALKLATE S. (Eds.), Handbook on sexual violence, xvii, 1, Oxon, Routledge.

KELLY L., 2019 [1989], Le continuum de la violence sexuelle, Cahiers du genre, Paris, $66,17-36$.

KENNEDY D., 2008, Sexy dressing.

Violences sexuelles et érotisation de la domination, Paris, Flammarion.

KISSLING E.A., 1991, Street harassment: the language of sexual terrorism, Discourse \& Society, 2, 4, 451-460.

LASCOUMES P., NAGELS C., 2014, Sociologie des élites délinquantes. De la criminalité en col blanc à la corruption politique, Paris, Armand Colin.

LEBUGLE A., 2018, Stéréotypes de genre et sexisme: principaux registres d'insultes dans les espaces publics, Cahiers $d u$ genre, 2, 65, 169-191.

LEBUGLE A. et l'équipe de l'enquête VIRAGE, 2017, Les violences dans les espaces publics touchent surtout les jeunes femmes des grandes villes, Population et sociétés, 150.

LIEBER M., 2008, Genre violences et espaces publics. La vulnérabilité des femmes en question. Presses de Sciences Po.

LOFLAND L.H, 1984, Women and Urban Public Space, Women and Environments, $6,2,12-14$.

LOGAN L.S., 2015, Street Harassment: Current and Promising Avenues for Researchers and Activists, Sociological Compass, 9, 3, 196-211.

MACKINNON C. A., 1979, Sexual Harassment of Working Women. A Case of Sex Discrimination, New Haven and London, Yale University Press.
MACMILLAN R., NIEROBISZ A., WELSH S., 2000, Experiencing the Streets: Harassment and Perceptions of Safety among Women, Journal of Research in Crime and Delinquency, 37, 3, 306-322.

MAILLARD J. (de), GAYET-VIAUD C., JOBARD F., MARET A., 2017, Aux bords de l'institution policière. Les délégués à la cohésion police-population (DCPP) en quête de crédibilité", Cahiers de la sécurité et de la justice, Paris, INHESJ.

MALBOIS F., 2011, Les catégories de sexe en action. Une sociologie praxéologique du genre, Sociologie, 1, 2, [en ligne] https://journals.openedition.org/ sociologie/876.

MCDOWELL L., 1999, Gender, Identity, and Place: Understanding Feminist Geographies, Minneapolis, University of Minnesota Press.

NIELSEN L.B., 2000, Situating Legal Consciousness. Experiences and Attitudes of Ordinary Citizens About Law and Street Harassment, Law and society review, 34, 4, 1055-1090.

NIELSEN L.B., 2009, License to Harass: Law, Hierarchy, and Offensive Public Speech, Princeton University Press.

NOBLE J., 2015, L'insécurité personnelle dans les transports en commun, Déviance et société, 3, 39, 343-364.

PAIN R., 2001, Gender, Race, Age and Fear in the City, Urban Studies, 38, 5/6, 899-913.

REGER J., 2015, The story of a slut walk: Sexuality, race, and generational divisions in contemporary feminist activism.

Journal of Contemporary ethnography, 44, 1, 84-112.

ROCHÉ S., 2002, Tolérance zéro? Incivilités et insécurité, Paris, Odile Jacob.

ROCHÉ S., 2000, Les règles de I'hospitalité dans les lieux collectifs, Hommes et migrations, 1127, 51-63. 
SERVICE STATISTIQUE MINISTÉRIEL DE LA SÉCURITÉ INTÉRIEURE (SSMI), 2018, Rapport d'enquête "Cadre de vie et sécurité». Victimation, délinquance et sentiment $d$ 'insécurité, [en ligne] https://www.interieur.gouv.fr/Interstats/ Actualites.

SKOGAN W.G., 1990, Disorder and Decline: Crime and the Spiral of Decay in American Neighborhoods, Berkeley, University of California Press.

SMITH D.E., 1987, The Everyday World as Problematic: A Feminist Sociology, Toronto, University of Toronto Press.

STEINBUGLER A., 2005, Visibility as Privilege and Danger: Heterosexual and Same-Sex Interracial Intimacy in the21st Century, Sexualities, 8, 4, 425-443.

STOP STREET HARASSMENT, 2014, Unsafe and Harassed in Public Spaces: A National Street Harassment Report, [en ligne] http://www.stopstreetharassment. org/wp-content/uploads/2012/08/

National-Street-Harassment-ReportNovember-29-20151.pdf.
VERA-GRAY F., 2016, Men's Intrusion, Women's Embodiment: A Critical Analysis of Street Harassment, London, Routledge.

VERA-GRAY F., 2018, The Right Amount of Panic: How Women Trade Freedom for Safety, Bristol, Policy Press.

WELSH S., CARR. J., MACQUARRIE B., HUNTLEY A., 2006. "I'm not thinking of it as sexual harassment": Understanding harassment across race and citizenship, Gender and Society, 20, 1, 87-107.

WESSELMANN E.D., KELLY J.R., 2010, Cat-calls and culpability: Investigating the frequency and Functions of Stranger harassment, Sex Roles, 63, 451-462. 
FR - Cet article revient sur la constitution du harcèlement de rue comme problème public en France, et sur la façon dont la notion de "continuum de violence" a été mobilisée au fil de ce processus. L'usage conjoint des notions de continuum et du harcèlement, appliquées aux formes de sexisme prenant place dans la rue, a permis la reconnaissance médiatique et politique d'un phénomène, via la publicisation d'une expérience féminine collective des rapports en public, marquée par un certain type d'offenses et d'atteintes à la liberté. Mais des difficultés se posent au moment d'envisager les réponses publiques apportées au problème ainsi configuré. La réflexion aujourd'hui ouverte sur le traitement politique et judiciaire du harcèlement de rue peut être éclairée par les enseignements issus des théories et politiques visant les incivilités: engagées en France et en Europe depuis les années 1990, elles se sont adossées à des conceptions de l'ordre public urbain, et de l'articulation entre formes mineures de déviance et criminalité, qui ont des traits communs avec les thèses aujourd'hui mobilisées pour justifier la pénalisation du harcèlement de rue. L'identification de ces analogies offre l'occasion d'une mise en perspective des bénéfices et des coûts qu'impliquent les approches aujourd'hui dominantes du harcèlement de rue, et de l'usage politique (et juridique) qui y est fait du concept de continuum de violence.

HARCÈLEMENT - CONTINUUM DE VIOLENCE ESPACES PUBLICS URBAINS - INCIVILITÉS THÉORIE DE LA VITRE BRISÉE - POLITIOUE PÉNALE

EN - This article revisits the constitution of street harassment as a public problem in France, and analyses more specifically how the ideas of "sexual harassment" and "continuum of violence" have been mobilised through this process. In the case of street harassment, these concepts have contributed to the construction of a "public" and a cause, by dramatising a series of gestures and words hitherto conceived as negligible. This dramatisation proved decisive in the process of politicisation (publicising a collective female experience) but it still raises difficulties when considering the public responses to the problem thus configured. We would like to nurture today's open reflections on the political and judicial treatment of street harassment, on the basis of the insights stemming from theories and policies aimed at incivilities. Prevalent in France (and elsewhere in Europe) from the 1990s, from the importation of North American models, these struggles have leaned towards conceptions of urban public order, and of the articulation between the 
minor forms of deviance and serious crime, which share several features with the ideas used to justify the criminalisation of street harassment. The identification of these analogies sheds light on both the benefits and costs of today's dominant approaches to street harassment, including the political (and legal) use of the concept of the continuum of violence.

HARASSMENT - VIOLENCE CONTINUUM -

URBAN PUBLIC SPACES - INCIVILITY -

BROKEN WINDOWS THEORY - CRIMINAL JUSTICE

DE - Dieser Beitrag greift die Thematik der sexuellen Belästigung im öffentlichen Raum als ein allgemeines Problem in Frankreich auf und zeigt, wie die Begriffe des "Gewaltkontinuums" und der „sexuellen Belästigung” verwendet wurden. Die gemeinsame Verwendung der Begriffe bezogen auf Formen sexistischer Handlungen im öffentlichen Raum, hat, mittels der Dramatisierung von Gesten und Worten, eine Öffentlichkeit hergestellt sowie die mediale und politische Anerkennung eines Phänomens ermöglicht. Auch wenn sich diese Dramatisierung im politischen Prozess als entscheidend herausstellte - die Öffentlichmachung der Erfahrung von Frauen in Berichten über bestimmte Straftaten und Angriffen auf die Freiheit -, so ergeben sich doch Schwierigkeiten. Lehren aus den Theorien und Strategien zu Verwahrlosungserscheinungen und Unordnung im öffentlichen Raum kann die Reflexion über den heute offeneren politischen und rechtlichen Umgang mit dem Thema der sexuellen Belästigung in der Öffentlichkeit fördern. Beginnend in den 1990er Jahren in Frankreich (und anderswo in Europa), auf der Grundlage des Imports nordamerikanischer Modelle, wurde dessen Bekämpfung an die Konzepte der öffentlichen Ordnung in den Städten angelehnt sowie an den Diskurs über Formen leichter und schwerer Kriminalität. Dieser weist Gemeinsamkeiten mit den Thesen auf, welche als Rechtfertigung der Sanktionierung sexueller Belästigung im öffentlichen Raum herangezogen werden. Das Aufzeigen der Analogien bietet die Möglichkeit, die Kosten und Nutzen der derzeit vorherrschenden Ansätze im Bereich der sexuellen Belästigung im öffentlichen Raum einander gegenüberzustellen, insbesondere den politischen (und rechtlichen) Gebrauch des Konzepts des Gewaltkontinuums.

BELÄSTIGUNG - GEWALTKONTINUUM STÄDTISCHER ÖFFENTLICHER RAUM ÖFFENTLICHE ORDNUNG - BROKEN-WINDOWS-THEORIE KRIMINALPOLITIK 
ES - Este artículo trata de la definición del acoso callejero como problema público en Francia y a cómo se ha movilizado en ese proceso la noción de "continuo de violencia». El uso conjunto de las nociones de continuo y de acoso, aplicadas a formas de sexismo en la calle, ha permitido el reconocimiento mediático y político de un fenómeno a través de la divulgación de una experiencia femenina colectiva de las relaciones en el ámbito público, marcada por cierto tipo de delitos y atentados a la libertad. Pero surgen dificultades a la hora de considerar las respuestas públicas al problema así configurado. La reflexión sobre el tratamiento político y judicial del acoso callejero puede aclararse con las lecciones aprendidas de las teorías y políticas sobre el comportamiento incívico: planteadas en Francia (y en Europa) desde la década de 1990 fueron respaldadas por las concepciones sobre el orden público urbano y la articulación entre formas menores de desviación y la criminalidad, que tienen rasgos con las tesis hoy movilizadas para justificar la criminalización del acoso callejero. La identificación de estas analogías nos permite poner en perspectiva los beneficios y costes de los enfoques actualmente dominantes sobre el acoso callejero y, el uso político (y legal) que se hace de él en el concepto de "continuum de la violencia».

ACOSO - CONTINUO DE VIOLENCIAS -

ESPACIOS PÚBLICOS URBANOS - INCIVISMO -

TEORÍA DE LAS VENTANAS ROTAS - PENALIZACIÓN 